\title{
JNS Review Recognition 2018
}

Journal of Nutritional Science (2018), vol. 7, e31, page 1 of 1

doi:10.1017/jns.2018.24

The Editorial Board of the Journal of Nutritional Science would like to thank the following for their contribution as peer reviewers in 2018.

Folasade Adebayo

Karen Assmann

Nuri Aykan

Claire Berryman

Maria Bouga

Kathryn Bradbury

Louise Brough

Aline Lopes Bueno

Ruud Buijs

Elisabet Børsheim

Maria Cavaletto

Ling-Wei Chen

Marike Cockeran

Vanessa de Mello Laaksonen

Alison Eldridge

Emily Farina

Emma Feeney

Michael Flock

Kurt Gedrich

Eileen Gibney

Ryan Grant

William Grant

Ingibjörg Gunnarsdóttir

Ella Haddad

Neil Harris

Christina Hartmann
Alyson Hill

Adela Hruby

Jasminka Ilich-Ernst

Geert Janssens

Hana Kahleova

Rajeev Kapila

Toshiyuki Kohri

Wojciech Krężel

Venu Lagishetty

Ashley Lederer

Alberto Leguina

Kenneth Lo

Bohdan Luhovyy

Kelsey Mangano

Koutatsu Maruyama

Thomas Mason

Anine Medin

Charlotte Mortensen

Ana Carolina Mosca

Kentaro Murakami

Sohail Mushtaq

Ran Nir-Paz

Camila Oliveira

Sue Penckofer

Stefany Primeaux

Gail Rees
Caroline Richard

Christian Ritz

Luis Romero

Mayra Saleh

Jay Schulkin

Barbara Korousic Seljak

Shigenobu Shibata

Barbara Shukitt-Hale

Kylie Smith

Christine Sommer

Matúš Soták

Xiong Su

Priya Sumithran

Izabela Szczerbal

Norman Temple

Sam Thiagalingam

Tammy Tong

Paul Trayhurn

Hans Van Trijp

Vaclav Vetvicka

Carol Wagner

Ben Wheeler

Philip Whitfield

Corné van Dooren

Linde van Lee

(C) The Author(s) 2018. This is an Open Access article, distributed under the terms of the Creative Commons Attribution licence (http://creativecommons.org/licenses/by/4.0/), which permits unrestricted re-use, distribution, and reproduction in any medium, provided the original work is properly cited. 